\title{
Stochastic Modelling of Wireless Energy Transfer
}

\author{
Shaun Veilleux, Ahmed Almaghasilah, Ali Abedi, DeLisa Wilkerson ${ }^{1}$ \\ Wireless Sensor Network (WiSe-Net) Lab \\ Electrical and Computer Engineering Department \\ ${ }^{1}$ Electronics Systems Branch \\ University of Maine, Orono, ME, USA \\ NASA Marshall Space Flight Center \\ Huntsville, AL, USA
}

\begin{abstract}
This study investigates the efficiency of a new method of powering remote sensors by the means of wireless energy transfer. The increased use of sensors for data collection comes with the inherent cost of supplying power from sources such as power cables or batteries. Wireless energy transfer technology eliminates the need for power cables or periodic battery replacement. The time and cost of setting up or expanding a sensor network will be reduced while allowing sensors to be placed in areas where running power cables or battery replacement is not feasible.

This paper models wireless channels for power and data separately. Smart scheduling for the data channel is proposed to avoid transmitting data on a noisy channel where the probability of data loss is high to improve power efficiency. Analytical models have been developed and verified using simulations.
\end{abstract}

\section{INTRODUCTION}

Wireless energy transfer will allow for sensors to receive power and transmit data without the use of the wires or the need to exchange batteries. This energy transmission method is ideal for sensors that have a low sampling rate, quick sampling time, and a varying sampling period. This technology is able to power multiple sensors at once and allow for changing the position of the sensor(s), and add additional sensors to the network with relative ease.

Technologies such as WiFi, cellular, and television (TV) that are considered abandoned wireless energy sources. They can be put in use to power and charge wireless sensors and batteries. For example, a TV signal is harvested and converted from a RF signal to DC using a RF-DC converter circuit, to power microcontroller and transceiver relay [1]. The wireless energy harvesting beacon system (WEH) is able to capture energy from the TV signal with a range of over $6.5 \mathrm{~km}$.

Technologies, such as microwave power transfer (MPT) and mobile communication offloading are designed to extend the mobile device's battery life [2]. The simultaneous wireless information and power transfer (SWIPT) field is a combination of two technologies with a goal of increasing the efficiency of the MPT.

This paper investigates the effects of smart scheduling on wireless sensors outage (the amount of time the sensor is not operational due to low energy). Smart scheduling is a method to determine when to transmit data based on the amount of stored energy in the sensor and the noise levels on the data channel. If there is too much noise on the wireless channel it is highly likely that the transmitted data will fail and be lost. If the sensor has low energy and the data channel has a high level of noise it would be best to preserve the energy and wait until either the sensor has more energy or the channel has less noise.

\section{Problem Formulation}

Research on wireless energy transfer will allow for sensors to receive energy and transmit data without the use of wires or the need to change batteries. As a wireless signal is transmitted it is reflected, scattered, and corrupted from the environment which prevents it from fully reaching the receiver. Three widely used channel models, AWGN, Rayleigh, and Rician are used to simulate the various cases detailed in Table I. The data and power are individually simulated through three channels. AWGN channel is suited for static charging situation, Rician channel is suited for mobile charging with a line of sight (LOS), and Rayleigh channel is used to model for mobile sensors with no dominant LOS.

A sensor takes time to receive and store enough energy to successfully transmit the sensor's data. The available energy for wireless energy transfer is limited, therefore, the implementation of smart scheduling is used to avoid wasting energy. If the noise on the data channel is above a threshold the sensor will not transmit or waste energy transmitting data that may not be received. Additionally, if the sensor's stored energy is below a threshold the sensor will not transmit to prevent an outage. For each unit of time, the power channel represents the amount of energy received. If the noise on the data channel is below a set threshold, the sensor will transmit.

\section{Simulation Results and Mathematical REPRESENTATIONS}

Simulations are modeled in MATLAB 2016b to display the effects of varying power and data channels between AWGN, Rayleigh, and Rician, detailed in Table I. The outage percentage versus the threshold with the inclusion of smart scheduling is represented in Figure 1. The first channel name corresponds to the power channel while the second is the data channel. If smart scheduling is not included the outage will be constant and equal to the max outage seen in Figure 1

It might not be possible to choose the environment the system is in, but the threshold can be chosen to maximize the 
TABLE I

MODELLED WIRELESS CHANNEL COMBINATIONS AND REAL WORLD EXAMPLES OF WHERE THEY MAY BE USED.

\begin{tabular}{|c|c|c|c|}
\hline Power/Data & AWGN & Rayleigh & Rician \\
\hline AWGN ${ }^{[1]}$ & Static sensor & Static charging for a mobile sensor & $\begin{array}{c}\text { Static charging for a mobile sensor with } \\
\text { LOS }^{[2]}\end{array}$ \\
\hline Rayleigh & Mobile charging for a static sensor & Mobile sensor & $\begin{array}{c}\text { Mobile charging for a mobile sensor } \\
\text { with LOS }\end{array}$ \\
\hline Rician & $\begin{array}{c}\text { Mobile charging with LOS for a static } \\
\text { sensor }\end{array}$ & $\begin{array}{c}\text { Mobile charging with LOS for a mobile } \\
\text { sensor }\end{array}$ & Mobile sensor with LOS \\
\hline
\end{tabular}

[1] AWGN - Additive White Gaussian Noise.

[2] LOS - Line of sight.

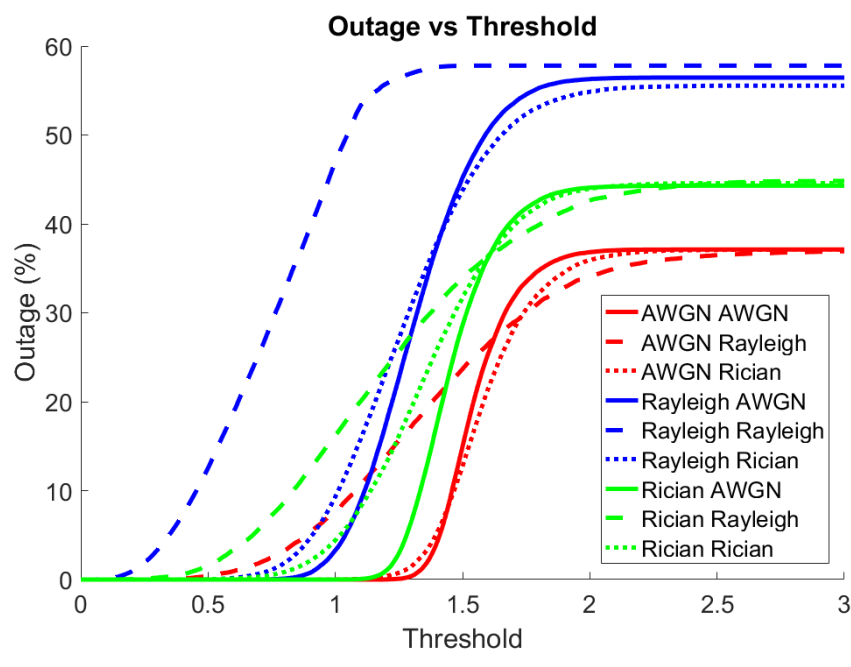

Fig. 1. The resultant outage vs threshold for modelled power channel and data channel

number of successful transmissions and minimize the outage. To choose the threshold a mathematical expression for the outage versus threshold is preferred over a simulation. When the threshold is low the sensor never transmits and therefore never uses energy, the result is an outage of zero percent. When the system has a high threshold the sensor will transmit every at time interval resulting in a maximum outage probability. To make the calculation of the max outage easier, the effects of the sensors energy storage will be ignored. Therefore the outage will be equal to the probability that the received power between each transmission is less than the required transmit power. For the case where the power channel is modeled using an AWGN channel the outage can be calculated using (1) (3), where (1) is the probability density function (PDF) of a normal distribution.

$$
N\left(\mu, \sigma^{2}\right)=\frac{1}{\sqrt{2 \pi \sigma^{2}}} e^{-\frac{(x-\mu)^{2}}{2 \sigma^{2}}}
$$

where $\mu$ is the mean and $\sigma$ is the variance of an AWGN channel.

$$
\sigma=\sqrt{10^{-\frac{S N R}{10}}}
$$

The outage is equal to the probability the received power is less than the power required to transmit, hence,

$$
\begin{aligned}
\text { outage } & =\int_{-\infty}^{x} N\left(\mu, \sigma^{2}\right) \\
& =\frac{1}{2}\left[1+\operatorname{erf}\left(\frac{x-\mu}{\sigma \sqrt{2}}\right)\right]
\end{aligned}
$$

where $\mathrm{x}$ is the power required to transmit. Simulation results represented in Figure 2 verify where the power channel is modeled using an AWGN channel, and the data channel is modeled using a Rayleigh channel.

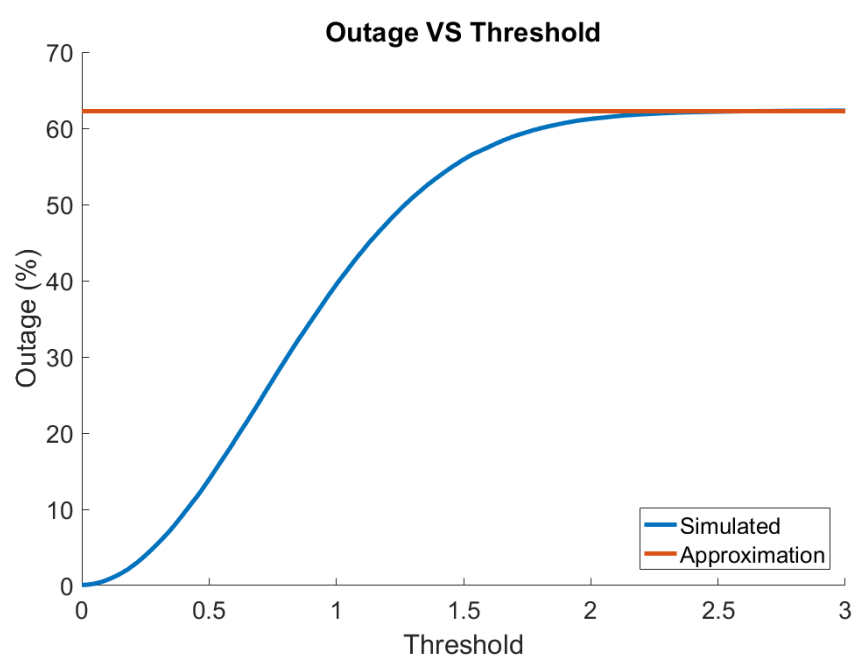

Fig. 2. The simulated outage vs the calculated max outage

As seen in Figure 2, calculating the maximum outage can be accurately accomplished using the cumulative density function (CDF) of the modeled power channel. Without the data channel and threshold taken into consideration the calculation is inaccurate when the threshold is not high. To calculate a better equation at any time interval, one needs to look at the energy received and amount needed to transmit. If the energy received is less than the amount needed to transmit and the noise on the data channel is less than the specified threshold, there will be an outage. Since the power channel and data are independent, the probability of an outage occurring are the probabilities multiplied together. For the case where the power channel is modelled using an AWGN channel, the data channel is modelled using a Rayleigh channel, the probability that the received energy is less than the energy needed to transmit is presented in (3). The probability that the noise on the data 
channel is less than the threshold can be calculated using (4) and (5).

$$
f(x, \sigma)=\frac{x}{\sigma^{2}} e^{\frac{-x}{2 \sigma^{2}}}
$$

where $\mathrm{x}$ is the threshold and $\sigma$ is the variance.

$$
\begin{aligned}
F(x, \sigma) & =\int_{0}^{x} f(x, \sigma) \\
& =1-e^{\frac{-x^{2}}{2 \sigma^{2}}}
\end{aligned}
$$

Therefore the total outage can be calculated as

$$
\begin{aligned}
\text { outage } & =\left(\int_{-\infty}^{x_{1}} N\left(\mu, \sigma_{1}^{2}\right)\right) *\left(F\left(x_{2}, \sigma_{2}^{2}\right)\right) \\
& =\frac{1}{2}\left[1+\operatorname{erf}\left(\frac{x_{1}-\mu}{\sigma_{1} \sqrt{2}}\right)\right] *\left(1-e^{\frac{-x_{2}^{2}}{2 \sigma_{2}^{2}}}\right)
\end{aligned}
$$

where $x_{1}$ is the required transmit energy and $x_{2}$ is the threshold. Simulation in Figure 3 verifies (6) and with a tighter maximum outage bound.

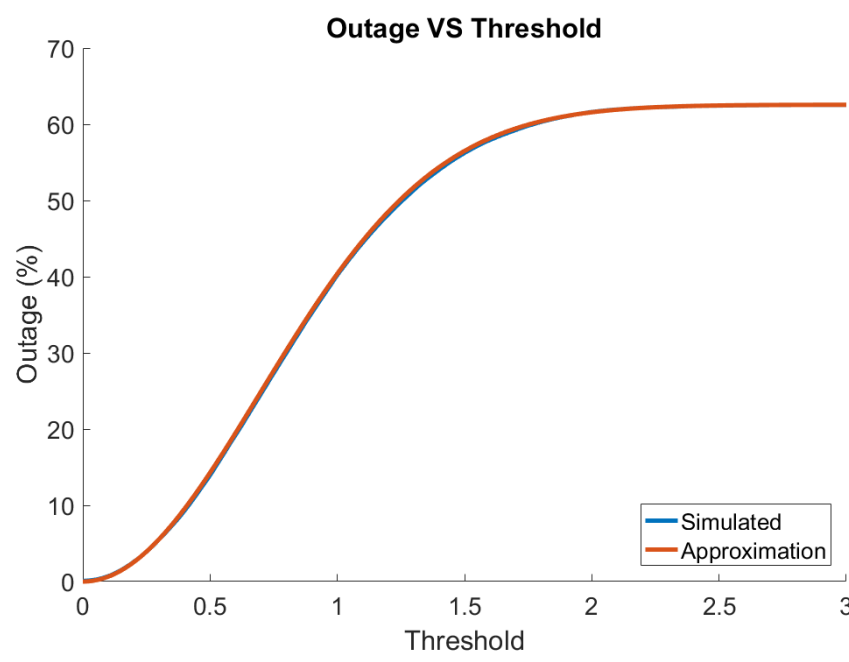

Fig. 3. The simulated outage vs the calculated max outage

The maximum outage bound calculated in (6) neglects the effects of the sensors energy storage. Figure 4 shows the simulation with the inclusion of the sensor energy storage, alongside the analytical result from (6).

\section{CONCLUSION}

With the increased use of sensors, it is desired to be able to decrease the setup time and maintenance cost. Wireless energy transfer technology helps achieve both of these. Sensors will no longer need expensive power cables or batteries to be periodically changed. This will allow for larger sensor networks to be established as well as reduction in time deployment. This paper focused on how to model a wireless stochastic network and the effects that smart scheduling has on a sensor's run time.

Three types of channels are investigated, AWGN, Rayleigh, and Rician as well and smart scheduling is examined to

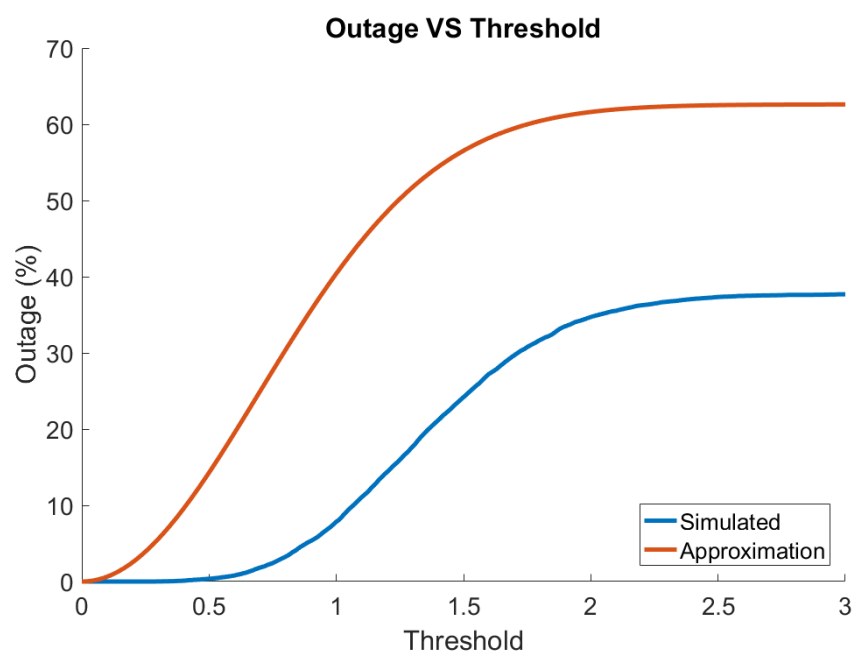

Fig. 4. The simulated outage with a battery vs the calculated max outage without a battery

decrease the outage percentage of a given sensor. Additionally, this paper has proposes a way to calculate the upper bound on the outage that a channel will see versus the threshold.

\section{REFERENCES}

[1] Hansen, R.C., J. McSpadden, and J.N. Benford. "A Universal Power Transfer Curve". IEEE Microwave and Wireless Components Letters 15.5 (2005): 369-371. Web. 1 Oct. 2016. [2] You, Changsheng, Kaibin Huang, and Hyukjin Chae. "Energy Efficient Mobile Cloud Computing Powered By Wireless Energy Transfer". IEEE Journal on Selected Areas in Communications 34.5 (2016): 1757-1771. Web. 1 Nov. 2016. [3] C. Bergsrud, S. Noghanian, J. Straub, D. Whalen and R. Fevig, "Orbit-to-ground Wireless Power Transfer test mission," 2013 IEEE Aerospace Conference, Big Sky, MT, 2013, pp. 111.

[4] C. R. Valenta and G. D. Durgin, "Harvesting Wireless Power: Survey of Energy-Harvester Conversion Efficiency in Far-Field, Wireless Power Transfer Systems," in IEEE Microwave Magazine, vol. 15, no. 4, pp. 108-120, June 2014. [5] D. Belo and N. B. Carvalho, "Harmonic spaced multisines for efficient wireless power transmission," 2015 IEEE Wireless Power Transfer Conference (WPTC), Boulder, CO, 2015, pp. $1-4$.

[6] D. Niyato, P. Wang and D. I. Kim, "Admission control policy for wireless networks with RF energy transfer," 2014 IEEE International Conference on Communications (ICC), Sydney, NSW, 2014, pp. 1118-1123.

[7] G. Wild and S. Hinckley, "Wireless acoustic communications and power supply for in vivo biomedical devices," 2010 IEEE International Ultrasonics Symposium, San Diego, CA, 2010, pp.

[8] A. Biason and M. Zorzi, "Joint Transmission and Energy Transfer Policies for Energy Harvesting Devices With Finite Batteries," in IEEE Journal on Selected Areas in Communications, vol. 33, no. 12, pp. 2626-2640, Dec. 2015. 